\title{
CLASSIFICATION OF GRADED LEFT-SYMMETRIC ALGEBRA STRUCTURES ON WITT AND VIRASORO ALGEBRAS
}

\author{
XIAOLI KONG, HONGJIA CHEN, AND CHENGMING BAI
}

\begin{abstract}
We find that a compatible graded left-symmetric algebra structure on the Witt algebra induces an indecomposable module of the Witt algebra with 1-dimensional weight spaces by its left multiplication operators. From the classification of such modules of the Witt algebra, the compatible graded left-symmetric algebra structures on the Witt algebra are classified. All of them are simple and they include the examples given by Chapoton and Kupershmidt. Furthermore, we classify the central extensions of these graded left-symmetric algebras which give the compatible graded left-symmetric algebra structures on the Virasoro algebra. They coincide with the examples given by Kupershmidt.
\end{abstract}

\section{INTRODUCTION}

Left-symmetric algebras (or under other names like pre-Lie algebras, quasi-associative algebras, Vinberg algebras and so on) are Lie-admissible algebras (see Proposition 2.2). They were introduced by A. Cayley in 1896 as a kind of rooted tree algebras ([Ca]). They also arose from the study of convex homogenous cones $([\mathrm{V}])$, affine manifolds and affine structures on Lie groups ([Ko] $)$, deformation of associative algebras $([\mathrm{G}])$ in 1960s. As it was pointed out in $[\mathrm{CL}$, the left-symmetric algebra "deserves more attention than it has been given". They appear in many fields of mathematics and mathematical physics. In $\mathrm{Bu} 2$, there is a survey of certain different fields where left-symmetric algebras play an important role, such as vector fields, rooted tree algebras, words in two letters, vertex algebras, operad theory, deformation complexes of algebras, convex homogeneous cones, affine manifolds, left-invariant affine structures on Lie groups. In addition, left-symmetric algebras have close relations with symplectic and complex structures on Lie groups and Lie algebras ([Chu], [LM], [DaM1-2], [AS]), phase spaces of Lie algebras ([Ku12], $[\mathrm{Ba}])$, certain integrable systems ([Bo], $[\mathrm{SS}]$ ), classical and quantum Yang-Baxter equations ([Ku3], [ES], GS], DiM] $)$, combinatorics $([\mathrm{E}]$ ) and so on. In particular, they play a crucial role in the Hopf algebraic approach of Connes and Kreimer to renormalization of perturbative quantum field theory $([\mathrm{CK}])$.

2000 Mathematics Subject Classification. 17B68, 17D25, 81R10.

Key words and phrases. Left-symmetric algebra, Witt algebra, Virasoro algebra. 
It is not easy to study left-symmetric algebras. Due to the nonassociativity, there is neither a suitable representation theory nor a complete structure theory like other classical algebras such as associative algebras and Lie algebras. For example, it is far from the classification of semisimple left-symmetric algebras (a left-symmetric algebra is called simple if it has not nontrivial ideals and a semisimple left-symmetric algebra is a direct sum of simple ones). In fact, the classification of complex simple left-symmetric algebras are only in low dimensions and some special cases in certain higher dimensions ([Bu1]).

On the other hand, an important approach to study left-symmetric algebras is through the representation theory of their sub-adjacent Lie algebras. It is known that there exists a compatible left-symmetric structure on a Lie algebra $\mathcal{G}$ if and only if $\mathcal{G}$ has an étale affine representation or equivalently, $\mathcal{G}$ has a bijective 1-cocycle associated to a representation (cf. [Me, [Ki]). Unfortunately, the sub-adjacent Lie algebra of a finite-dimensional left-symmetric algebra over a field of characteristic zero cannot be semisimple. So the beautiful representation theory of finitedimensional semisimple Lie algebras cannot be used here. However, in the case of the infinite dimensional, there exists a semisimple Lie algebra with a compatible left-symmetric algebra structure. One of such examples is given as a left-symmetric Witt algebra, which is regarded as the first important example of infinite-dimensional left-symmetric algebras (it was also regarded as one of the origins of left-symmetric algebras in [Bu2]). Let $U$ be an associative commutative algebra, and $\mathcal{D}=\left\{\partial_{1}, \cdots, \partial_{n}\right\}$ be a system of commutating derivations of $U$. Then the vector space

$$
\operatorname{Vec}(n)=\left\{\sum_{i=1}^{n} u_{i} \partial_{i} \mid u_{i} \in U, \partial_{i} \in \mathcal{D}\right\},
$$

is called a left-symmetric Witt algebra under the multiplication ([Ku1], [DL], [Bu2])

$$
u \partial_{i} \circ v \partial_{j}=u \partial_{i}(v) \partial_{j}
$$

In particular, when $n=1, U=\mathbb{F}\left[x, x^{-1}\right]$ and $\partial=\frac{\partial}{\partial x}$, the $W^{1}=\operatorname{Vec}(1)$ is a simple left-symmetric algebra, whose sub-adjacent Lie algebra is the Witt algebra.

Therefore, it is natural to consider the classification of the compatible left-symmetric algebra structures on the Witt algebra. It is easy to know that all compatible left-symmetric algebras on the Witt algebra are simple. Since the Witt algebra is graded, it is also natural to suppose that the compatible left-symmetric algebras should be graded. Hence, in this paper, we mainly consider the left-symmetric algebras with a basis $\left\{x_{n} \mid n \in \mathbb{Z}\right\}$ satisfying

$$
x_{i} x_{j}=f(i, j) x_{i+j}, \quad\left[x_{i}, x_{j}\right]=x_{i} x_{j}-x_{j} x_{i}=(j-i) x_{i+j},
$$


where $f$ is a complex-value function on $\mathbb{Z} \times \mathbb{Z}$. In particular, $W^{1}$ is just the case $f(i, j)=1+j$ and it is a Novikov algebra, which is a left-symmetric algebra with commutative right multiplication operators $([\mathrm{GD}],[\mathrm{BN}])$.

Moreover, like $W^{1}$, some other special cases satisfying equation (1.3) have been already discussed. For example, Chapoton in [Cha] gave the classification in the case $f(i, j)=g(i) h(j)$ (up to a change of basis). As it was pointed out in [Cha], "this Ansatz for the product has no special meaning, except that it allows for a full classification". Kupershmidt gave a solution $f(i, j)=\frac{j(1+b j)}{1+b(i+j)}$ for $b=0$ or $b^{-1} \notin \mathbb{Z}$ in [Ku2]. Osborn classified a class of simple infinite dimensional Novikov algebras which includes a classification of Novikov algebras satisfying equation (1.3), that is, $f(i, j)=\alpha+j$ for any $\operatorname{Re} \alpha>0$ or $\operatorname{Re} \alpha=0, \operatorname{Im} \alpha \geq 0([\mathrm{O})$. However, to our knowledge, a complete classification of left-symmetric algebras satisfying equation (1.3) is still unknown.

In this paper, we will mainly use the representation theory of the Witt algebra. We find that a regular representation of the Witt algebra induced by a compatible left-symmetric algebra structure satisfying equation (1.3) (through its left multiplication operators) belongs to a class of important modules of the Witt algebra, which have been classified. Hence such left-symmetric algebras can be classified through studying the relations between them. As it was done in Lie algebras, it is natural to consider the central extensions of the left-symmetric algebras satisfying equation (1.3), by which we can get the compatible left-symmetric algebra structures on the Virasoro algebra. It is interesting to see that there exist only one class of non-trivial central extensions which coincides with a result in $\mathrm{Ku} 2$.

We would like to point out some interesting remarks from the following aspects.

- Our methods in this paper provide an approach to the study of the possible compatible left-symmetric algebra structures on the infinite-dimensional (semi)simple Lie algebras with a good representation theory.

- The left-symmetric algebras obtained in this paper are the simple graded left-symmetric algebras of growth one. It is difficult to classify all of it. Our study can be regarded as the first step, as Mathieu solved the analogous problem for Lie algebras ([Ma1-3]).

- It will be also interesting to consider their relations with the vertex (operator) algebras which are the fundamental algebraic structures in conformal field theory, since a vertex algebra is equivalent to a left-symmetric algebra and a Lie conformal algebra with some compatible conditions $([\mathrm{BK}],[\mathrm{LL}])$. 
This paper is organized as follows. In Section 2, we give some necessary definitions, notations and basic results on left-symmetric algebras and the representation theory of the Witt and Virasoro algebras. In Section 3, we prove that a left-symmetric algebra structure satisfying equation (1.3) induces an indecomposable module of the Witt algebra with 1-dimensional weight spaces by its left multiplication operators. Therefore, such left-symmetric algebras are classified through the classification of those modules. In Section 4, the non-trivial central extensions of the left-symmetric algebras obtained in Section 3 are discussed. A classification of the compatible left-symmetric algebra structures on the Virasoro algebras are obtained.

Throughout this paper, all algebras are over the complex field $\mathbb{C}$ and the indices $m, n, l, i, j, k \in$ $\mathbb{Z}$, unless otherwise stated.

\section{Preliminaries and fundamental Results}

Definition 2.1. Let $A$ be a vector space over a field $\mathbb{F}$ equipped with a bilinear product $(x, y) \rightarrow x y . A$ is called a left-symmetric algebra if for any $x, y, z \in A$, the associator

$$
(x, y, z)=(x y) z-x(y z)
$$

is symmetric in $x, y$, that is,

$$
(x, y, z)=(y, x, z), \text { or equivalently }(x y) z-x(y z)=(y x) z-y(x z) .
$$

Left-symmetric algebras are Lie-admissible algebras (cf. [Me]).

Proposition 2.2. Let $A$ be a left-symmetric algebra. For any $x \in A$, let $L_{x}$ denote the left multiplication operator, that is, $L_{x}(y)=x y$ for any $y \in A$. Then we have the following results:

(1) The commutator

$$
[x, y]=x y-y x, \quad \forall x, y \in A,
$$

defines a Lie algebra $\mathcal{G}(A)$, which is called a sub-adjacent Lie algebra of $A$ and $A$ is also called a compatible left-symmetric algebra structure on the Lie algebra $\mathcal{G}(A)$.

(2) Let $L: \mathcal{G}(A) \rightarrow g l(A)$ with $x \mapsto L_{x}$. Then $(L, A)$ gives a representation of the Lie algebra $\mathcal{G}(A)$, that is,

$$
\left[L_{x}, L_{y}\right]=L_{[x, y]}, \quad \forall x, y \in A .
$$

We call it a regular representation of the Lie algebra $\mathcal{G}(A)$.

There is not a compatible left-symmetric algebra structure on any Lie algebra $\mathcal{G}$. A sufficient and necessary condition for a Lie algebra with a compatible left-symmetric algebra structure is 
given as follows. Let $\mathcal{G}$ be a Lie algebra and $\rho: \mathcal{G} \rightarrow g l(V)$ be a representation of $\mathcal{G}$. A 1-cocycle $q: \mathcal{G} \rightarrow V$, the linear map on vector space associated to $\rho$ (denoted by $(\rho, q)$ ) satisfying

$$
q[x, y]=\rho(x) q(y)-\rho(y) q(x), \forall x, y \in \mathcal{G} .
$$

Let $A$ be a left-symmetric algebra and $\rho: \mathcal{G}(A) \rightarrow g l(V)$ be a representation of its sub-adjacent Lie algebra. If $g$ is a homomorphism of the representations from $A$ to $V$, then $g$ is a 1-cocycle of $\mathcal{G}(A)$ associated to $\rho$.

Proposition 2.3. Let $\mathcal{G}$ be a Lie algebra. Then there is a compatible left-symmetric algebra structure on $\mathcal{G}$ if and only if there exists a bijective 1-cocycle of $\mathcal{G}$.

In fact, let $(\rho, q)$ be a bijective 1-cocycle of $\mathcal{G}$, then

$$
x * y=q^{-1} \rho(x) q(y), \quad \forall x, y \in \mathcal{G},
$$

defines a left-symmetric algebra structure on $\mathcal{G}$. Conversely, for a left-symmetric algebra $A$, the identity transformation $i d$ is a 1-cocycle of $\mathcal{G}(A)$ associated to the regular representation $L$.

On the other hand, we recall the definition of the Witt algebra $W$ and Virasoro algebra $\mathscr{V}$. The Witt algebra $W$ (of rank one) is a complex Lie algebra with a basis $\left\{x_{n} \mid n \in \mathbb{Z}\right\}$ whose commutation relations satisfy

$$
\left[x_{m}, x_{n}\right]=(n-m) x_{m+n} .
$$

The Virasoro algebra $\mathscr{V}$ is the central extension of $W$ defined by the 2-cocycle

$$
\Omega\left(x_{m}, x_{n}\right)=\frac{1}{12}\left(n^{3}-n\right) \delta_{m+n, 0} .
$$

That is, $\mathscr{V}$ is a complex Lie algebra with a basis $\left\{\theta, x_{n} \mid n \in \mathbb{Z}\right\}$ whose commutation relations satisfy

$$
\left[\theta, x_{n}\right]=0, \quad\left[x_{m}, x_{n}\right]=(n-m) x_{m+n}+\delta_{m+n, 0} \frac{n^{3}-n}{12} \theta .
$$

Since an ideal of a left-symmetric algebra is still an ideal of its sub-adjacent Lie algebra and $W$ is a simple Lie algebra, we have the following conclusion.

Proposition 2.4. Any compatible left-symmetric algebra structure on the Witt algebra $W$ is simple.

For any module $V$ of a Lie algebra $\mathcal{G}$, the action of $\mathcal{G}$ on $V$ is denoted by $x v$ for any $x \in \mathcal{G}$ and $v \in V$. Recall that a module $V$ of a Lie algebra is called indecomposable if $V$ cannot be decomposed into a direct sum of two proper submodules. A weight space of a module $V$ of the Witt algebra $W$ or the Virasoro algebra $\mathscr{V}$ is the non-zero vector space $V_{\lambda}$ defined by

$$
V_{\lambda}=\left\{x \in V \mid x_{0} v=\lambda v\right\}
$$


For later use, we give the description of the indecomposable modules with 1-dimensional weight spaces of the Virasoro algebra $\mathscr{V}([\mathrm{KS}],[\mathrm{MP} 1-2]$, [Ma4] $)$.

- The $\mathscr{V}$-module $A_{\alpha, \beta}$ of Feigin-Fuchs with $\alpha, \beta \in \mathbb{C}$ and $0 \leq \operatorname{Re} \alpha<1$, whose action on a basis $\left\{v_{n} \mid n \in \mathbb{Z}\right\}$ is given by

$$
x_{i} v_{n}=(\alpha+n+i \beta) v_{n+i}, \quad \theta v_{n}=0 .
$$

- The maximal proper $\mathscr{V}$-submodule of $A_{0,1}$, called $A_{0,1}^{\prime}=A_{0,1} \backslash \mathbb{C} v_{0} \quad\left(A_{0,1} / A_{0,1}^{\prime}\right.$ is trivial and $\left.A_{0,0} / \mathbb{C} v_{0} \simeq A_{0,1}^{\prime}\right)$, whose action on a basis $\left\{v_{n} \mid n \in \mathbb{Z} \backslash\{0\}\right\}$ is given by

$$
x_{i} v_{n}=(n+i) v_{n+i}, \quad \theta v_{n}=0, \quad \forall n \neq 0 .
$$

- The $\mathscr{V}$-module $A_{\alpha}$ with $\alpha \in \mathbb{C}$ whose action on a basis $\left\{v_{n} \mid n \in \mathbb{Z}\right\}$ is given by

$$
\begin{aligned}
& x_{i} v_{n}=(n+i) v_{n+i}, \quad \forall n \neq 0, \\
& x_{i} v_{0}=i(\alpha+i) v_{i}, \quad \theta v_{n}=0 .
\end{aligned}
$$

- The $\mathscr{V}$-module $B_{\beta}$ with $\beta \in \mathbb{C}$ whose action on a basis $\left\{v_{n} \mid n \in \mathbb{Z}\right\}$ is given by

$$
\begin{aligned}
& x_{i} v_{n}=n v_{n+i}, \quad \text { if } n+i \neq 0, \\
& x_{i} v_{-i}=-i(\beta+i) v_{0}, \quad \theta v_{n}=0 .
\end{aligned}
$$

Theorem 2.5. ([KS] $)$ Any indecomposable nontrivial module of the Virasoro algebra $\mathscr{V}$ with 1-dimensional weight spaces is isomorphic to one of $A_{\alpha, \beta}, A_{0,1}^{\prime}, A_{\alpha}, B_{\beta}$.

Obviously, $A_{\alpha, \beta}, A_{0,1}^{\prime}, A_{\alpha}$, and $B_{\beta}$ are also the modules of the Witt algebra $W$.

Corollary 2.6. Any indecomposable nontrivial module of the Witt algebra $W$ with 1dimensional weight spaces is isomorphic to one of $A_{\alpha, \beta}, A_{0,1}^{\prime}, A_{\alpha}, B_{\beta}$.

\section{Compatible left-Symmetric algebra structures on the Witt Algebra}

As we said in the introduction, we study the following compatible left-symmetric algebra structure on the Witt algebra $W$ with the multiplication

$$
x_{m} x_{n}=f(m, n) x_{m+n},
$$

where $\left\{x_{n} \mid n \in \mathbb{Z}\right\}$ is a basis of $W$ satisfying equation (2.7). To avoid confusion, we denote such a left-symmetric algebra structure by $V$. Then $V$ is a regular module of $W$ defined by the left multiplication operators of $V$, that is, in equation (3.1), we let $x_{m} \in W$ and $x_{n}, x_{m+n} \in V$.

In fact, $V$ is a compatible left-symmetric algebra structure on $W$ if and only if

$$
\left[x_{m}, x_{n}\right]=x_{m} x_{n}-x_{n} x_{m}=(n-m) x_{m+n} \text { and }\left(x_{m}, x_{n}, x_{l}\right)=\left(x_{n}, x_{m}, x_{l}\right) .
$$


They hold if and only if $f(m, n)$ satisfies the following equations:

$$
\begin{gathered}
f(m, n)-f(n, m)=n-m, \\
(n-m) f(m+n, l)=f(n, l) f(m, n+l)-f(m, l) f(n, m+l) .
\end{gathered}
$$

Lemma 3.1. $f(m, 0)=f(0,0)$.

Proof. Let $n=l=0$ in equation (3.4), we have

$$
-m f(m, 0)=f(0,0) f(m, 0)-f(m, 0) f(0, m) .
$$

Then by equation (3.3), we have

$$
f(m, 0)(f(0, m)-m-f(0,0))=f(m, 0)(f(m, 0)-f(0,0))=0 .
$$

Therefore, $f(m, 0)=0$ or $f(m, 0)=f(0,0)$.

If $f(0,0)=0$, then $f(m, 0)=0=f(0,0)$. The conclusion holds.

If $f(0,0) \neq 0$, set

$$
I_{1}=\{m \in \mathbb{Z} \mid f(m, 0)=0\}, I_{2}=\{m \in \mathbb{Z} \mid f(m, 0)=f(0,0)\} .
$$

Obviously, $I_{1} \cup I_{2}=\mathbb{Z}, I_{1} \cap I_{2}=\emptyset$ and $0 \in I_{2}$.

Let $l=0$ in equation (3.4), we have

$$
(n-m) f(m+n, 0)=f(n, 0) f(m, n)-f(m, 0) f(n, m) .
$$

Then we obtain the following results:

(a) If $m, n \in I_{s}, m \neq n(s=1,2)$, then $m+n \in I_{s}$;

(b) If $m \in I_{1}$, then $-m \in I_{2}$.

Next we prove that $1,-1,2,-2 \notin I_{1}$, that is $1,-1,2,-2 \in I_{2}$. Thus by the above result (a), it is easy to know that $I_{2}=\mathbb{Z}$ and $I_{1}=\emptyset$. The conclusion holds.

(I). $1 \notin I_{1}$.

Otherwise, we suppose $1 \in I_{1}$. Hence $-1 \in I_{2}, 2 \in I_{1}$ (if $2 \in I_{2}$, then $1=2+(-1) \in I_{2}$ which is a contradiction). Therefore, for any $n>0$, we know $n \in I_{1}$ by induction. Thus

$$
I_{1}=\{1,2, \ldots\}, \quad I_{2}=\{0,-1,-2, \ldots\} .
$$

If $-n \geq m>0$, then equation $(*)$ becomes

$$
(n-m) f(0,0)=f(0,0) f(m, n) .
$$

Hence $f(m, n)=n-m, f(n, m)=0$. In particular, if $n<0$, we have $f(n, 1)=0$. 
If $m>-n>0$, then $m+n>0$, and equation $(*)$ becomes

$$
f(0,0) f(m, n)=0 .
$$

Hence $f(m, n)=0, f(n, m)=m-n$. In particular, if $m \geq-n>0, f(n, m+1)=m+1-n$.

Let $l=1$ in equation (3.4), we have

$$
(n-m) f(m+n, 1)=f(n, 1) f(m, n+1)-f(m, 1) f(n, m+1) .
$$

Therefore when $m \geq-n>0$, the above equation becomes

$$
(n-m) f(m+n, 1)=(n-m-1) f(m, 1) .
$$

Let $m=-n>0$. Then we have

$$
2 m f(0,1)=(2 m+1) f(m, 1), \quad m \geq 1,
$$

where $f(0,1)=1$ since $1 \in I_{1}$ and $f(1,0)=0$.

Let $m=1-n>1$. Then we have

$$
(2 m-1) f(1,1)=2 m f(m, 1), \quad m \geq 2 .
$$

Thus, by the above two equations, we know that

$$
f(1,1)=\frac{4 m^{2}}{4 m^{2}-1}
$$

for all $m \geq 2$, which is impossible.

(II). $-1 \notin I_{1}$. The proof is similar to the proof in Case (I) by symmetry.

(III). $2 \notin I_{1}$.

Otherwise suppose $2 \in I_{1}$. Therefore $1,0,-1,-2 \in I_{2}$. Now for any $n \geq 2$, we have $n \in I_{1}$ by induction since $n=(n+1)+(-1)$. Thus

$$
I_{1}=\{2,3, \ldots\}, I_{2}=\{1,0,-1,-2, \ldots\} .
$$

If $m-1>-n>0$, that is, $m+n>1, m>1$, then equation $(*)$ becomes

$$
0=f(0,0) f(m, n)
$$

Hence $f(m, n)=0, f(n, m)=m-n$. In particular, if $m \geq-n \geq 2$, then we have $f(m+2, n)=$ $0, f(n, m+2)=m+2-n$.

If $-m \geq n>1$, then equation $(*)$ becomes

$$
(n-m) f(0,0)=-f(0,0) f(n, m) .
$$

Hence $f(n, m)=m-n, f(m, n)=0$. In particular, if $n \leq-2$, then $f(n, 2)=0$. 
Let $l=2$ in equation (3.4), we have

$$
(n-m) f(m+n, 2)=f(n, 2) f(m, n+2)-f(m, 2) f(n, m+2) .
$$

Therefore, when $m \geq-n \geq 2$, the above equation becomes

$$
(m-n) f(m+n, 2)=(m+2-n) f(m, 2) .
$$

Let $m=-n \geq 2$. Then we have

$$
2 m f(0,2)=(2 m+2) f(m, 2), \quad m \geq 2,
$$

where $f(0,2)=2$ since $2 \in I_{1}$ and $f(2,0)=0$.

Let $m=1-n \geq 3$. Then we have

$$
(2 m-1) f(1,2)=(2 m+1) f(m, 2), \quad m \geq 3 .
$$

Thus, by the above two equations, we know that

$$
f(1,2)=\frac{4 m^{2}+2 m}{2 m^{2}+m-1}
$$

for all $m \geq 3$, which is impossible.

Case (IV). $-2 \notin I_{1}$. The proof is similar to the proof in Case (III) by symmetry.

Lemma 3.2. The weight space of $V$ is 1-dimensional.

Proof. By Lemma 3.1 and equation (3.3), we have $f(0, m)=f(0,0)+m$, that is, the elements $\left\{x_{n}\right\}$ are in different eigenspaces of $x_{0}$. So every weight space of $V$ is 1-dimensional.

Lemma 3.3. $V$ is an indecomposable $W$-module.

Proof. We assume that $V=V_{1} \oplus V_{2}$, where $V_{1}, V_{2}$ are proper submodules of $V$. We assume that $V_{2}$ is indecomposable and $x_{0} \notin V_{2}$ without losing generality. It is well known that any submodule of a weight module is still a weight module ([Ka]). Then there exist two nonempty subsets $I_{1}, I_{2}$ of $\mathbb{Z}$, such that

$$
0 \notin I_{2}, \quad I_{1} \cup I_{2}=\mathbb{Z}, \quad I_{1} \cap I_{2}=\emptyset, \quad \text { and } V_{i}=\bigoplus_{n \in I_{i}} \mathbb{C} x_{n}, \quad i=1,2 .
$$

Let $s$ be the smallest positive integer in $I_{2}$ (if all elements of $I_{2}$ are negative, then let $s$ be the largest negative integer, and the discussion is similar). We have the following results.

(i) $f(m, 0)=0, f(0, m)=m$. In fact, $x_{s} x_{0}=f(s, 0) x_{s} \in V_{1} \cap V_{2}$ since $x_{0} \in V_{1}$. So $f(s, 0)=0$. Therefore we have $f(m, 0)=f(s, 0)=0$ by Lemma 3.1.

(ii) If $m, n \in I_{2}$ and $m \neq n$, then $m+n \in I_{2}$. In fact, since $f(m, n)-f(n, m)=n-m \neq 0$, at least one of $f(m, n), f(n, m)$ is not zero. Suppose $f(m, n) \neq 0$ without losing generality. Then $x_{m} x_{n}=f(m, n) x_{m+n} \neq 0$. Hence $x_{m+n} \in V_{2}$. 
(iii) If $m \in I_{2}$, then $-m \in I_{1}$. Otherwise, by (ii), we know that $0=m+(-m) \in I_{2}$, which contradicts to our assumption.

(iv) If $m \in I_{k}(k=1,2), f(n, m) \neq 0$, then $m+n \in I_{k}$.

(v) If $m \in I_{2}$ and $n \in I_{1}$, then $m+n \in I_{1}$ if and only if $f(n, m)=0, f(m, n)=n-m$ and $m+n \in I_{2}$ if and only if $f(m, n)=0, f(n, m)=m-n$.

Both (iv) and (v) immediately follow from $x_{n} x_{m}=f(n, m) x_{m+n}$.

(vi) $s+1 \in I_{2}$. In fact, let $-m=n=1$ and $l=s$ in equation (3.4), we have,

$$
2 f(0, s)=f(1, s) f(-1, s+1)-f(-1, s) f(1, s-1) .
$$

By (i), $f(0, s)=s$. Since $s-1 \in I_{1}$ due to our assumption on $s, f(-1, s)=0$ by (iv). Therefore we know that $2 s=f(1, s) f(-1, s+1) \neq 0$. Hence $f(1, s) \neq 0$. So $s+1 \in I_{2}$ by (iv).

If $s=1$, then $2 \in I_{2}$. Moreover, $\{n \in \mathbb{Z} \mid n \geq 1\} \subset I_{2}$ by (ii) and $\{n \in \mathbb{Z} \mid n \leq 0\} \subset I_{1}$ by (iii). That is,

$$
I_{1}=\{0,-1,-2, \ldots\}, \quad I_{2}=\{1,2, \ldots\} .
$$

By equation $(* *)$ and $(\mathrm{v})$, we know that $f(1,1)=\frac{2}{3}$. Let $m=-n=2$ and $l=s=1$ in equation (3.4), we know that $f(2,1)=\frac{4}{5}$. Let $m=2, n=-1, l=s=1$ in equation (3.4), we have

$$
-3 f(1,1)=f(-1,1) f(2,0)-f(2,1) f(-1,3) .
$$

Therefore, $2=\frac{16}{5}$, which is a contradiction.

Now supposing that $s>1$, we have $x_{s-1} \in V_{1}, x_{1} \in V_{1}$. Hence $x_{1} x_{s-1} \in V_{1} \cap V_{2}$ and $x_{s-1} x_{1} \in$ $V_{1} \cap V_{2}$. Therefore $f(1, s-1)=f(s-1,1)=0$. Since $f(1, s-1)-f(s-1,1)=s-2$ by equation (3.3), we know that $s-2=0$, that is, $s=2$. Moreover, $-1 \in I_{1}$ by (ii), $-2 \in I_{1}$ by (iii) and $3 \in I_{2}$ by (vi). By equation (**) and (v), we know that $f(1,2)=1$. Let $m=-n=2, l=s=2$ in equation (3.4), we have

$$
-4 f(0,2)=f(-2,2) f(2,0)-f(2,2) f(-2,4) .
$$

By (i), $f(0,2)=2$ and $f(2,0)=0$. Hence $f(2,2) \neq 0$. Then $4=2+2 \in I_{2}$ by (iv). Furthermore, by $(\mathrm{v})$, we know that $f(-2,4)=6$. Therefore $f(2,2)=\frac{4}{3}$. Let $m=2, n=-1, l=s=2$ in equation (3.4), we have

$$
-3 f(1,2)=f(-1,2) f(2,1)-f(2,2) f(-1,4) .
$$

Therefore, $3=\frac{20}{3}$, which is a contradiction, too.

Thus there does not exist such an $s$ and hence $V$ is an indecomposable $W$-module.

Corollary 3.4. As a $W$-module, $V$ must be isomorphic to one of $A_{\alpha, \beta}, A_{0,1}^{\prime}, A_{\alpha}, B_{\beta}$. 
Next we discuss the existence and the classification of the left-symmetric algebras $V$.

Theorem 3.5. As a $W$-modules, $V$ is not isomorphic to $A_{0,1}^{\prime}$.

Proof. Suppose that $V \cong A_{0,1}^{\prime}$, where $V$ is given by the multiplication $x_{m} x_{n}=f(m, n) x_{m+n}$. Then there exists an isomorphism $g: V \rightarrow A_{0,1}^{\prime}$ of modules such that $g\left(x_{0}\right)=\sum_{i \neq 0} c_{i} v_{i}$, for a finite number of nonzero $c_{i} \in \mathbb{C}$. Since $g\left(x_{0} x_{0}\right)=x_{0} g\left(x_{0}\right)$, we have

$$
\sum_{i \neq 0} f(0,0) c_{i} v_{i}=\sum_{i \neq 0} c_{i} i v_{i}
$$

Because $g$ is an isomorphism, there exists $k \neq 0$, such that $c_{k} \neq 0$ and $f(0,0)=k$. Then $g\left(x_{0}\right)=c_{k} v_{k}$ and by Lemma 3.1, $f(m, 0)=k$. Since $g\left(x_{-k} x_{0}\right)=x_{-k} g\left(x_{0}\right)$, we know that $f(-k, 0) g\left(x_{-k}\right)=0$. Therefore $g\left(x_{-k}\right)=0$, which is a contradiction.

By a similar discussion as above, we have the following conclusion.

Theorem 3.6. As a $W$-modules, $V$ is not isomorphic to $A_{\alpha}$.

Theorem 3.7. There are compatible left-symmetric algebra structures $V_{\alpha, \epsilon}$ on the Witt algebra $W$ given by the multiplication

$$
x_{m} x_{n}=\frac{(\alpha+n+\alpha \epsilon m)(1+\epsilon n)}{1+\epsilon(m+n)} x_{m+n},
$$

where $\alpha, \epsilon \in \mathbb{C}$ satisfying $\epsilon=0$ or $\epsilon^{-1} \notin \mathbb{Z}$.

Proof. Suppose that $V \cong A_{\alpha, \beta}$, where $\alpha, \beta \in \mathbb{C}, 0 \leq \operatorname{Re} \alpha<1$, and $V$ is given by the multiplication $x_{m} x_{n}=f(m, n) x_{m+n}$. Then there exists an isomorphism $g: V \rightarrow A_{\alpha, \beta}$ of modules such that $g\left(x_{0}\right)=\sum_{i} c_{i} v_{i}$ for a finite number of nonzero $c_{i} \in \mathbb{C}$. Since $g\left(x_{0} x_{0}\right)=x_{0} g\left(x_{0}\right)$, we have

$$
\sum_{i} f(0,0) c_{i} v_{i}=\sum_{i} c_{i}(\alpha+i) v_{i}
$$

So there exists $k$ such that $c_{k} \neq 0$ and $f(0,0)=\alpha+k$. Then $g\left(x_{0}\right)=c_{k} v_{k}$ and by Lemma 3.1, $f(m, 0)=\alpha+k$. Since $g\left(x_{m} x_{0}\right)=x_{m} g\left(x_{0}\right)$, we know that

$$
f(m, 0) g\left(x_{m}\right)=c_{k} x_{m} v_{k}=c_{k}(\alpha+k+m \beta) v_{m+k} .
$$

If $\alpha+k \neq 0$, then $g\left(x_{m}\right)=\frac{\alpha+k+m \beta}{\alpha+k} c_{k} v_{m+k}$. Moreover, in this case, $\alpha+k+m \beta \neq 0$ since $g$ is an isomorphism. Since $g\left(x_{m} x_{n}\right)=x_{m} g\left(x_{n}\right)$, we have

$$
f(m, n) g\left(x_{m+n}\right)=\frac{\alpha+k+n \beta}{\alpha+k} c_{k} x_{m} v_{n+k}=\frac{\alpha+k+n \beta}{\alpha+k}(\alpha+n+k+m \beta) c_{k} v_{m+n+k} .
$$

Therefore,

$$
f(m, n)=\frac{(\alpha+k+n+m \beta)(\alpha+k+n \beta)}{\alpha+k+(m+n) \beta} .
$$


Replacing $\alpha+k$ and $\frac{\beta}{\alpha+k}$ by $\alpha$ and $\epsilon$ respectively, we have

$$
f(m, n)=\frac{(\alpha+n+\alpha \epsilon m)(1+\epsilon n)}{1+\epsilon(m+n)},
$$

where $\alpha \neq 0$ and $\epsilon=0$ or $\epsilon^{-1} \notin \mathbb{Z}$.

If $\alpha+k=0$, then $\alpha=k=0$ since $0 \leq \operatorname{Re} \alpha<1$. Hence $m \beta c_{k} v_{m+k}=0$. Thus $\beta=0$. Moreover, we have $f(m, 0)=0$ and $f(0, m)=m$. Since $m g\left(x_{m}\right)=g\left(x_{0} x_{m}\right)=x_{0} g\left(x_{m}\right)$, we know that $g\left(x_{m}\right)=a_{m} v_{m}$, where $a_{m} \neq 0$. In particular, $a_{0}=c_{0}$. Since

$$
f(m, n) a_{m+n} v_{m+n}=f(m, n) g\left(x_{m+n}\right)=g\left(x_{m} x_{n}\right)=x_{m} g\left(x_{n}\right)=n a_{n} v_{m+n},
$$

we know that $f(m, n)=\frac{n a_{n}}{a_{m+n}}$. By equation (3.3), we have

$$
(n-m) a_{m+n}=n a_{n}-m a_{m}
$$

Let $m=-n$, we have $a_{n}+a_{-n}=2 a_{0}$. On the other hand, let $m=2, n=-1$, we have $a_{-1}=3 a_{1}-2 a_{2}$. Therefore $a_{2}=2 a_{1}-a_{0}$. By induction, we know that $a_{n}=n a_{1}-(n-1) a_{0}$. Replacing $\frac{a_{1}-a_{0}}{a_{0}}$ by $\epsilon$, we have

$$
f(m, n)=\frac{n a_{n}}{a_{m+n}}=\frac{n\left(n a_{1}-n a_{0}+a_{0}\right)}{(m+n) a_{1}-(m+n) a_{0}+a_{0}}=\frac{n(1+n \epsilon)}{1+\epsilon(m+n)},
$$

where $\epsilon=0$ or $\epsilon^{-1} \notin \mathbb{Z}$. Moreover, it is just the case $\alpha=0$ in equation (3.5).

Conversely, it is easy to know that we define a compatible left-symmetric algebra structure on the Witt algebra $W$ by equation (3.5).

Theorem 3.8. There are compatible left-symmetric algebra structures $V^{\beta, k}$ on the Witt algebra $W$ given by the multiplications

$$
\begin{gathered}
x_{m} x_{n}=(n+k) x_{m+n}, \text { if } m+n+k \neq 0, \\
x_{-n-k} x_{n}=\frac{(n+k)(\beta-n-k)}{\beta-k} x_{-k},
\end{gathered}
$$

where $\beta \in \mathbb{C}$ and $k \in \mathbb{Z}$ satisfying $\beta-k \neq 0$.

Proof. Suppose that $V \cong B_{\beta}$, where $\beta \in \mathbb{C}$, and $V$ is given by the multiplication $x_{m} x_{n}=$ $f(m, n) x_{m+n}$. Then there exists an isomorphism $g: V \rightarrow B_{\beta}$ of modules such that $g\left(x_{0}\right)=$ $\sum_{i} c_{i} v_{i}$ for a finite number of nonzero $c_{i} \in \mathbb{C}$. Since $g\left(x_{0} x_{0}\right)=x_{0} g\left(x_{0}\right)$, we have

$$
\sum_{i} f(0,0) c_{i} v_{i}=\sum_{i \neq 0} c_{i} i v_{i}
$$

So there exists $k$ such that $c_{k} \neq 0$ and $f(0,0)=k$. Then $g\left(x_{0}\right)=c_{k} v_{k}$ and by Lemma 3.1, $f(m, 0)=k$. 
Case (I). $k \neq 0$. Since $g\left(x_{m} x_{0}\right)=x_{m} g\left(x_{0}\right)$, we have

$$
f(m, 0) g\left(x_{m}\right)=c_{k} k v_{m+k}, \quad \text { if } m+k \neq 0 ; \quad f(m, 0) g\left(x_{-k}\right)=c_{k} k(\beta-k) v_{0} .
$$

Hence $g\left(x_{m}\right)=c_{k} v_{m+k}$, when $m \neq-k$ and $g\left(x_{-k}\right)=c_{k}(\beta-k) v_{0}$. In this case, $\beta-k \neq 0$ since $g$ is an isomorphism. Since $g\left(x_{m} x_{n}\right)=x_{m} g\left(x_{n}\right)$, we have

$$
\begin{gathered}
f(m, n) g\left(x_{m+n}\right)=c_{k} x_{m} v_{n+k} \text { if } n+k \neq 0, \\
f(m,-k) g\left(x_{m-k}\right)=c_{k}(\beta-k) x_{m} v_{0}=0 .
\end{gathered}
$$

Case (I-1). $m+n+k \neq 0$. If $n \neq-k$, we have

$$
f(m, n) c_{k} v_{m+n+k}=c_{k} x_{m} v_{n+k}=(n+k) c_{k} v_{m+n+k} .
$$

Thus $f(m, n)=n+k$. If $n=-k$, we have $f(m,-k)=0$. Therefore we know that

$$
f(m, n)=n+k, \text { for all } m, n \in \mathbb{Z} \text { with } m+n+k \neq 0 .
$$

Case (I-2). $m+n+k=0$. If $n \neq-k$, we have

$$
f(-k-n, n) c_{k}(\beta-k) v_{0}=c_{k} x_{m} v_{n+k}=(n+k)(\beta-n-k) c_{k} v_{0}
$$

Then $f(-k-n, n)=\frac{(n+k)(\beta-n-k)}{\beta-k}$. If $n=-k$, we have $f(0,-k)=0$. Therefore we know that

$$
f(m, n)=\frac{(n+k)(\beta-n-k)}{\beta-k}, \text { for all } m, n \in \mathbb{Z} \text { with } m+n+k=0 .
$$

Case (II). $k=0$. Then $f(0, m)=m$. Since

$$
x_{0} g\left(x_{m}\right)=g\left(x_{0} x_{m}\right)=f(0, m) g\left(x_{m}\right)=m g\left(x_{m}\right),
$$

we know that $g\left(x_{m}\right)=d_{m} v_{m}$, where $d_{m} \neq 0$. In particular, $d_{0}=c_{0}$. Since

$$
(n-m) d_{m+n} v_{m+n}=g\left(\left[x_{m}, x_{n}\right]\right)=x_{m} g\left(x_{n}\right)-x_{n} g\left(x_{m}\right)=d_{n} x_{m} v_{n}-d_{m} x_{n} v_{m}
$$

we have

$$
(n-m) d_{m+n}=n d_{n}-m d_{m} \text {, if } m+n \neq 0 ; \quad(\beta-n) d_{n}+(\beta+n) d_{-n}=2 d_{0} .
$$

Let $m=2$ and $n=-1$, we have $d_{-1}=3 d_{1}-2 d_{2}$. Let $m=-2$ and $n=1$, we have $d_{-2}=4 d_{1}-3 d_{2}$. On the other hand, let $n=1$, we have $(\beta-1) d_{1}+(\beta+1)\left(3 d_{1}-2 d_{2}\right)=2 d_{0}$. Let $n=2$, we have $(\beta-2) d_{2}+(\beta+2)\left(4 d_{1}-3 d_{2}\right)=2 d_{0}$. Therefore, we know that

$$
d_{0}=\beta d_{1}, \quad d_{1}=d_{2}=d_{-1}=d_{-2} .
$$

By induction, we have $d_{n}=d_{1}$ for all $n \neq 0$ and $d_{0}=\beta d_{1}$, where $\beta \neq 0$ since $d_{0} \neq 0$. Because

$$
f(m, n) g\left(x_{m+n}\right)=g\left(x_{m} x_{n}\right)=x_{m} g\left(x_{n}\right),
$$


with a similar discussion as in Case (I), we know that

$$
f(m, n)=\left\{\begin{array}{cl}
n, & \text { if } m+n \neq 0 ; \\
\frac{n(\beta-n)}{\beta}, & \text { if } m+n=0, \text { where } \beta \neq 0 .
\end{array}\right.
$$

Obviously, it is just the case $k=0$ in equations (3.6) and (3.7).

Conversely, it is easy to know that we define a compatible left-symmetric algebra structure on the Witt algebra $W$ by equations (3.6) and (3.7).

Lemma 3.9. Let $T$ be an automorphism of the Witt algebra $W$. Then $T\left(x_{0}\right)= \pm x_{0}$.

Proof. Suppose $T\left(x_{0}\right)=\sum_{i} c_{i} x_{i}$ and $T\left(x_{m}\right)=\sum_{j} d_{j} x_{j}$ for a finite number of nonzero $c_{i}, d_{j} \in \mathbb{C}$. Therefore we have

$$
m \sum_{j} d_{j} x_{j}=T\left(\left[x_{0}, x_{m}\right]\right)=\left[T\left(x_{0}\right), T\left(x_{m}\right)\right]=\sum_{i+j=l}(j-i) c_{i} d_{j} x_{l} .
$$

If there exists $i>0$ such that $c_{i} \neq 0$, then let $n$ and $k$ be the maximal numbers in the sets $\left\{i \mid c_{i} \neq 0\right\}$ and $\left\{j \mid d_{j} \neq 0\right\}$ respectively. Therefore, by comparing the coefficient of $x_{n+k}$ in both hand sides of the above equation, we have $0=(k-n) c_{n} d_{k}$. So $k=n$. However, since $m$ is arbitrary, we know that $\operatorname{Im} T \subset \bigoplus_{i \leq n} \mathbb{C} x_{i}$, which is contradictory to the assumption that $T$ is an automorphism of $W$. By a similar discussion, we can prove that there does not exist $i<0$ such that $c_{i} \neq 0$.

Therefore $T\left(x_{0}\right)=c_{0} x_{0}$. Since $m \sum_{j} d_{j} x_{j}=\sum_{j} j c_{0} d_{j} x_{j}$, we know that there exists $k$ such that $d_{k} \neq 0$ and $m=k c_{0}$ for any $m \neq 0$. Hence $T\left(x_{m}\right)=d_{k} x_{k}$. In particular, there exists $m_{1} \in \mathbb{Z}$, such that $T\left(x_{m_{1}}\right)=d_{1} x_{1}$. So $c_{0}=m_{1}$. Moreover, since $c_{0} \mid m$ for any $m \in \mathbb{Z}$, we have $c_{0}= \pm 1$.

Proposition 3.10. Let $\left(V_{1}, \cdot\right)$ and $\left(V_{2}, *\right)$ be two compatible left-symmetric algebras structures on the Witt algebra $W$ defined by

$$
x_{m} \cdot x_{n}=f_{1}(m, n) x_{m+n}, \quad \text { and } \quad x_{m} * x_{n}=f_{2}(m, n) x_{m+n}
$$

respectively. If $V_{1}$ is isomorphic to $V_{2}$ as left-symmetric algebras, then

$$
f_{1}(m, n)=f_{2}(m, n) \text {, or } f_{1}(m, n)=-f_{2}(-m,-n) .
$$

Proof. Let $T$ be an isomorphism from $V_{1}$ into $V_{2}$, that is, $T(x \cdot y)=T(x) * T(y)$ for all $x, y \in V_{1}$. Obviously, $T$ is an automorphism of $W$. By Lemma 3.9, we have $T\left(x_{0}\right)= \pm x_{0}$.

If $T\left(x_{0}\right)=x_{0}$, then there exists $a_{m} \neq 0$ such that $T\left(x_{m}\right)=a_{m} x_{m}$ from the proof of Lemma 3.9. Hence it is obvious that $a_{m} a_{n}=a_{m+n}$ and $a_{0}=1$. Since

$$
f_{1}(m, n) a_{m+n} x_{m+n}=T\left(x_{m} \cdot x_{n}\right)=T\left(x_{m}\right) * T\left(x_{n}\right)=f_{2}(m, n) a_{m} a_{n} x_{m+n},
$$


we have $f_{1}(m, n)=f_{2}(m, n)$.

If $T\left(x_{0}\right)=-x_{0}$, then there exists $b_{m} \neq 0$ such that $T\left(x_{m}\right)=b_{m} x_{-m}$ from the proof of Lemma 3.9. Hence it is easy to know that $b_{m} b_{n}=-b_{m+n}$ and $b_{0}=-1$. Since

$$
f_{1}(m, n) b_{m+n} x_{-(m+n)}=T\left(x_{m} \cdot x_{n}\right)=T\left(x_{m}\right) * T\left(x_{n}\right)=f_{2}(-m,-n) b_{m} b_{n} x_{-(m+n)},
$$

we have $f_{1}(m, n)=-f_{2}(-m,-n)$.

Theorem 3.11. Any graded compatible left-symmetric algebra structure on the Witt algebra $W$ satisfying equation (3.1) is isomorphic to one of the following algebras:

$$
\begin{aligned}
& V_{\alpha, \epsilon}, \quad \alpha, \epsilon \in \mathbb{C} \text { satisfying } \epsilon=0 \text { or } \epsilon^{-1} \notin \mathbb{Z} ; \\
& V^{\beta, k}, \quad \beta \in \mathbb{C} \text { and } k \in \mathbb{Z} \text { satisfying } \beta \neq k .
\end{aligned}
$$

Moreover, the isomorphisms between them are exactly given as follows,

$$
V_{\alpha, \epsilon} \cong V_{-\alpha,-\epsilon}, \quad V_{\alpha, 0} \cong V_{\alpha, 1 / \alpha} \text { with } \alpha \notin \mathbb{Z} \text { and } V^{\beta, 0} \cong V^{-\beta, 0}
$$

Proof. The first half part immediately follows from Corollary 3.4 and Theorems 3.5-3.8. For the second half part, we assume that $V_{1} \cong V_{2}$, where $V_{1}, V_{2}$ are the compatible left-symmetric algebra structures on $W$ defined by equation $(3.1)$ with $f_{1}(m, n)$ and $f_{2}(m, n)$ respectively. Then by Proposition 3.10, we have $f_{1}(m, n)=f_{2}(m, n)$ or $f_{1}(m, n)=-f_{2}(-m,-n)$. Obviously, in the former case, $V_{1}=V_{2}$ and in the latter case, $V_{1} \cong V_{2}$ by the linear isomorphism $x_{n} \rightarrow-x_{-n}$.

Case (I). $V_{1}=V_{\alpha_{1}, \epsilon_{1}}$ and $V_{2}=V_{\alpha_{2}, \epsilon_{2}}$.

If $f_{1}(m, n)=f_{2}(m, n)$, then $f_{1}(0,0)=\alpha_{1}=f_{2}(0,0)=\alpha_{2}$. Set $\alpha_{1}=\alpha_{2}=\alpha$. Since

$$
f_{1}(m, n)=\frac{\left(\alpha+n+\alpha \epsilon_{1} m\right)\left(1+\epsilon_{1} n\right)}{1+\epsilon_{1}(m+n)}=\frac{\left(\alpha+n+\alpha \epsilon_{2} m\right)\left(1+\epsilon_{2} n\right)}{1+\epsilon_{2}(m+n)}=f_{2}(m, n),
$$

we have

$$
\left(\epsilon_{1}-\epsilon_{2}\right)\left[\alpha\left(\epsilon_{1}+\epsilon_{2}\right)-1+\alpha \epsilon_{1} \epsilon_{2}(m+n)\right]=0 .
$$

If $\alpha=0$, then $\epsilon_{1}=\epsilon_{2}$. In this case, $V_{1}=V_{2}=V_{0, \epsilon}$. If $\alpha \neq 0$, then $\epsilon_{1}=\epsilon_{2}$ or $\epsilon_{1}=0, \epsilon_{2}=\frac{1}{\alpha}$ or $\epsilon_{2}=0, \epsilon_{1}=\frac{1}{\alpha}$. Therefore, we have $V_{1}=V_{2}=V_{\alpha, \epsilon_{1}}$, or

$$
V_{1}=V_{\alpha, 0}, \quad V_{2}=V_{\alpha, 1 / \alpha}, \alpha \notin \mathbb{Z} ; \quad \text { or } V_{1}=V_{\alpha, 1 / \alpha}, V_{2}=V_{\alpha, 0}, \quad \alpha \notin \mathbb{Z} .
$$

If $f_{1}(m, n)=-f_{2}(-m,-n)$, then $f_{1}(0,0)=\alpha_{1}=-f_{2}(0,0)=-\alpha_{2}$. Set $\alpha_{1}=-\alpha_{2}=\alpha$. Since

$$
f_{1}(m, n)=\frac{\left(\alpha+n+\alpha \epsilon_{1} m\right)\left(1+\epsilon_{1} n\right)}{1+\epsilon_{1}(m+n)}=\frac{\left(\alpha+n-\alpha \epsilon_{2} m\right)\left(1-\epsilon_{2} n\right)}{1-\epsilon_{2}(m+n)}=-f_{2}(-m,-n),
$$

we have

$$
\left(\epsilon_{1}+\epsilon_{2}\right)\left[\alpha\left(\epsilon_{1}-\epsilon_{2}\right)-1-\alpha \epsilon_{1} \epsilon_{2}(m+n)\right]=0
$$


If $\alpha=0$, then $\epsilon_{1}=-\epsilon_{2}$. In this case, $V_{1}=V_{0, \epsilon} \cong V_{2}=V_{0,-\epsilon}$. If $\alpha \neq 0$, then $\epsilon_{1}=-\epsilon_{2}$ or $\epsilon_{1}=0$, $\epsilon_{2}=-\frac{1}{\alpha}$ or $\epsilon_{2}=0, \epsilon_{1}=\frac{1}{\alpha}$. Therefore, we have

$$
\begin{gathered}
V_{1}=V_{\alpha, \epsilon}, V_{2}=V_{-\alpha,-\epsilon} ; \text { or } V_{1}=V_{\alpha, 0}, V_{2}=V_{-\alpha,-1 / \alpha}, \alpha \notin \mathbb{Z} ; \\
\text { or } V_{1}=V_{\alpha, 1 / \alpha}, V_{2}=V_{-\alpha, 0}, \alpha \notin \mathbb{Z} .
\end{gathered}
$$

Case (II). $V_{1}=V^{\beta_{1}, k_{1}}$ and $V_{2}=V^{\beta_{2}, k_{2}}$.

If $f_{1}(m, n)=f_{2}(m, n)$, then $f_{1}(0,0)=k_{1}=f_{2}(0,0)=k_{2}$. Set $k_{1}=k_{2}=k$. Since

$$
f_{1}(-n-k, n)=\frac{(n+k)\left(\beta_{1}-n-k\right)}{\beta_{1}-k}=\frac{(n+k)\left(\beta_{2}-n-k\right)}{\beta_{2}-k}=f_{2}(-n-k, n),
$$

we know that $\beta_{1}=\beta_{2}$.

If $f_{1}(m, n)=-f_{2}(-m,-n)$, then $f_{1}(0,0)=k_{1}=-f_{2}(0,0)=-k_{2}$. Set $k_{1}=-k_{2}=k$. If $k \neq 0$, then let $n \neq 0,-k$. Since $f_{1}(-n-k, n)=-f_{2}(n+k,-n)$, we have

$$
\frac{(n+k)\left(\beta_{1}-n-k\right)}{\beta_{1}-k}=-(-n-k) .
$$

So $\beta_{1}-n-k=\beta_{1}-k$, which is a contradiction. Therefore $k=0$. Since

$$
f_{1}(-n, n)=\frac{n\left(\beta_{1}-n\right)}{\beta_{1}}=-\frac{-n\left(\beta_{2}+n\right)}{\beta_{2}}=-f_{2}(n,-n),
$$

we have $\beta_{1}=-\beta_{2}$. On the other hand, when $\beta_{1}=-\beta_{2}=\beta$ and $k_{1}=k_{2}=0$, it is easy to know that $f_{1}(m, n)=-f_{2}(-m,-n)$ and then $V_{1}=V^{\beta, 0} \cong V_{2}=V^{-\beta, 0}$.

Case (III). $V_{1}=V_{\alpha, \epsilon}$ and $V_{2}=V^{\beta, k}$.

If $f_{1}(m, n)=f_{2}(m, n)$, then $f_{1}(0,0)=\alpha=f_{2}(0,0)=k \in \mathbb{Z}$. Obviously there exist $m, n$ such that $m \neq 0, n \neq 0$ and $m+n+k \neq 0$. Since

$$
f_{1}(m, n)=\frac{(k+n+k \epsilon m)(1+\epsilon n)}{1+\epsilon(m+n)}=n+k=f_{2}(m, n),
$$

we have

$$
\epsilon m n(k \epsilon-1)=0 .
$$

Since $\epsilon^{-1} \notin \mathbb{Z}$, we know $k \epsilon-1 \neq 0$. Then $\epsilon=0$. Let $n \neq 0,-k$. Since

$$
f_{1}(-n-k, n)=k+n=\frac{(k+n)(\beta-k-n)}{\beta-k}=f_{2}(-n-k, n),
$$

we have $\beta-k-n=\beta-k$, which is a contradiction.

If $f_{1}(m, n)=-f_{2}(-m,-n)$, then we still have $f_{1}(m, n)=f_{2}(m, n)$ by taking $V_{1}=V_{-\alpha,-\epsilon}$ and $V_{2}=V^{\beta, k}$. Since $V_{\alpha, \epsilon} \cong V_{-\alpha,-\epsilon}$, we know that $V_{\alpha, \epsilon}$ is not isomorphic to $V^{\beta, k}$ in this subcase.

So there does not exist an isomorphism between $V_{\alpha, \epsilon}$ and $V^{\beta, k}$.

Example 3.12. In Cha], the notion of pre-Lie algebra was used. Chapoton classified the simple graded left-symmetric algebras of growth one satisfying the following two conditions: 
(1) The underlying graded vector space is $E=\oplus_{i \in \mathbb{Z}} \mathbb{C} e_{i}$;

(2) The product is given by $e_{i} \circ e_{j}=f(i) g(j) e_{i+j}$.

Such a left-symmetric algebra is isomorphic either to the algebra $A_{a}$ defined by

$$
e_{i} \circ e_{j}=(1+a j) e_{i+j}, \quad a \in \mathbb{C},
$$

or to the algebra $B_{b}$ defined by

$$
e_{i} \circ e_{j}=\frac{j}{1+b i} e_{i+j}, \quad b=0 \text { or } b^{-1} \notin \mathbb{Z} .
$$

Obviously, $A_{0}$ is a commutative associative algebra and $B_{0} \cong V_{0,0}$. Moreover, when $a \neq 0$, $A_{a} \cong V_{\frac{1}{a}, 0}$ by a linear transformation $e_{i} \rightarrow \frac{1}{a} e_{i}$. When $b \neq 0, b^{-1} \notin \mathbb{Z}, B_{b} \cong V_{0, b}$ by a linear transformation $e_{i} \rightarrow(1+b i) e_{i}$. Therefore, the only isomorphisms among $A_{a}$ and $B_{b}$ are $A_{a} \cong A_{-a}$ and $B_{b} \cong B_{-b}$ due to Theorem 3.11, which were given in [Cha], too. Hence, except $A_{0}$, we have obtained all left-symmetric algebras in [Cha].

In particular, $V_{0, b}$ for $b=0$ or $b^{-1} \notin \mathbb{Z}$ was also given in [Ku2] as a special case of the left-symmetric algebras satisfying equation (3.1).

Example 3.13. Recall that a Novikov algebra $A$ is a left-symmetric algebra satisfying $(x y) z=(x z) y$ for any $x, y, z \in A$. Novikov algebras have been introduced in connection with Hamiltonian operators in the formal variational calculus (GD]) and Poisson brackets of hydrodynamic type $([\mathrm{BN}])$. It is easy to see that a compatible Novikov algebra structure on the Witt algebra satisfying equation (3.1) must be isomorphic to one of $N_{\alpha}=V_{\alpha, 0}$, that is,

$$
x_{m} x_{n}=(\alpha+n) x_{m+n}, \quad \alpha \in \mathbb{C} .
$$

Moreover, the isomorphisms between them are exactly given by $N_{\alpha} \cong N_{-\alpha}$. It is just the case (ii) appearing in $\mathrm{O}$.

\section{Compatible left-Symmetric algebra structures on the Virasoro algebra}

In this section, we consider the central extensions of the left-symmetric algebras obtained in Section 3 whose commutator is the Virasoro algebra $\mathscr{V}$.

Let $(A, \cdot)$ be a left-symmetric algebra and $\omega: A \times A \rightarrow \mathbb{C}$ be a bilinear form. It defines a multiplication on the space $\widehat{A}=A \oplus \mathbb{C} \theta$, by the rule

$$
(x+a \theta) *(y+b \theta)=x \cdot y+\omega(x, y) \theta, \quad x, y \in A, a, b \in \mathbb{C} .
$$

Obviously, $\widehat{A}$ is a left-symmetric algebra if and only if

$$
\omega(x \cdot y, z)-\omega(x, y \cdot z)=\omega(y \cdot x, z)-\omega(y, x \cdot z) .
$$


$\widehat{A}$ is called a central extension of $A$. Moreover, by construction, the bilinear form

$$
\Omega(x, y)=\omega(x, y)-\omega(y, x), \quad \text { where } x, y \in \mathcal{G}(A)
$$

defines a central extension of its sub-adjacent Lie algebra $\mathcal{G}(A)$.

Let $V$ be a compatible left-symmetric algebra structure on the Witt algebra $W$ satisfying equation (3.1). Since the Virasoro algebra $\mathscr{V}$ is a central extension of the Witt algebra $W$, it is natural to consider the central extension $\widehat{V}=V \oplus \mathbb{C} \theta$ of $V$ such that $\widehat{V}$ is a compatible left-symmetric algebra structure on the Virasoro algebra $\mathscr{V}$ while $\theta$ being the annihilator of $\mathscr{V}$, that is, the products are given by

$$
\theta \theta=x_{m} \theta=\theta x_{m}=0 \text { and } x_{m} x_{n}=f(m, n) x_{m+n}+\omega\left(x_{m}, x_{n}\right) \theta
$$

where $f(m, n)$ satisfies equations (3.3)-(3.4).

We denote $\omega\left(x_{m}, x_{n}\right)$ by $\omega(m, n)$ for convenience. Then by equations (2.8), (4.2) and (4.3), we have the following equations

$$
\begin{gathered}
\omega(m, n)-\omega(n, m)=\frac{1}{12}\left(n^{3}-n\right) \delta_{m+n, 0}, \\
(n-m) \omega(m+n, l)=\omega(m, n+l) f(n, l)-\omega(n, m+l) f(m, l) .
\end{gathered}
$$

Theorem 4.1. When $\alpha \neq 0$ or $\epsilon=0$, there does not exist a central extension of $V_{\alpha, \epsilon}$ satisfying equations (4.5)-(4.6). There is exactly one central extension of $V_{0, \epsilon}$ with $\epsilon \neq 0$ and $\epsilon^{-1} \notin \mathbb{Z}$ satisfying equations (4.5)-(4.6), which is given by:

$$
\omega\left(x_{m}, x_{n}\right)=\omega(m, n)=\frac{1}{24}\left(n^{3}-n-\left(\epsilon-\epsilon^{-1}\right) n^{2}\right) \delta_{m+n, 0} .
$$

Proof. Let $m=-n \neq 0, l=0$ in equation (4.6), we have

$$
\omega(0,0)=\frac{1}{24} \alpha\left(n^{2}-1\right)
$$

Since $\omega(0,0)$ does not depend on $n$, we know that $\alpha=0$ and $\omega(0,0)=0$. Notice that $V_{0, \epsilon}$ is given by (see $[\mathrm{Ku} 2]$, too)

$$
f(m, n)=\frac{n(1+\epsilon n)}{1+\epsilon(m+n)} .
$$

Let $n=l=0$ in equations (4.5) and (4.6), then we get

$$
\omega(m, 0)=\omega(0, m)=0
$$

Let $m=0$ in equation (4.6), thus we know

$$
n \omega(n, l)=\omega(0, n+l) f(n, l)-\omega(n, l) f(0, l)=-l \omega(n, l),
$$


that is, $(n+l) \omega(n, l)=0$. So we can assume that

$$
\omega(n, l)=\varphi(n) \delta_{n+l, 0} \text { for some map } \varphi: \mathbb{Z} \rightarrow \mathbb{C} .
$$

Let $m+n=0$ in equation (4.5), thereby we have

$$
\varphi(n)-\varphi(-n)=\frac{1}{12}\left(-n^{3}+n\right)
$$

Let $m+n+l=0$ in equation (4.6), then

$$
(n-m) \varphi(m+n)=\varphi(m) f(n,-m-n)-\varphi(n) f(m,-m-n),
$$

which gives

$$
(n-m) \varphi(m+n)=\frac{(-m-n)(1-\epsilon(m+n))}{1-\epsilon m} \varphi(m)-\frac{(-m-n)(1-\epsilon(m+n))}{1-\epsilon n} \varphi(n) .
$$

Set $\psi(m)=\frac{\varphi(m)}{m(1-\epsilon m)}$. Then we know that

$$
\left\{\begin{array}{l}
(1-\epsilon n) \psi(n)+(1+\epsilon n) \psi(-n)=\frac{1}{12}\left(-n^{2}+1\right), \\
(n-m) \psi(m+n)=-m \psi(m)+n \psi(n) .
\end{array}\right.
$$

Let $m=2, n=-1$ in equation $(* * *)$, so we have

$$
(1+\epsilon) \psi(-1)+(1-\epsilon) \psi(1)=0,-3 \psi(1)=-2 \psi(2)-\psi(-1) .
$$

So $\psi(2)=\frac{\epsilon+2}{\epsilon+1} \psi(1)$. Let $m=-2, n=1$ in the first part of equation $(* * *)$, hence we get

$$
3 \psi(-1)=2 \psi(-2)+\psi(1) .
$$

So $\psi(-2)=\frac{\epsilon-2}{\epsilon+1} \psi(1)$. Notice that (by the first part of equation $(* * *)$ )

$$
(1-2 \epsilon) \psi(2)+(1+2 \epsilon) \psi(-2)=-\frac{1}{4} .
$$

If $\epsilon=0$, then

$$
\psi(2)+\psi(-2)=2 \psi(1)-2 \psi(1)=0=-\frac{1}{4},
$$

which is a contradiction. So we suppose that $\epsilon \neq 0$. Then $\psi(1)=\frac{1}{24}\left(1+\epsilon^{-1}\right)$. Let $n=1$ in the second part of equation $(* * *)$, we have

$$
(1-m) \psi(m+1)=-m \psi(m)+\psi(1) .
$$

Then

$$
(m-1)(\psi(m+1)-\psi(1))=m(\psi(m)-\psi(1)) .
$$

Without losing generality, we can assume that $m \geq 2$ (a similar discussion is for $m \leq 0$ ). Therefore,

$$
\psi(m+1)-\psi(1)=\frac{m}{m-1} \cdot \frac{m-1}{m-2} \cdots \frac{2}{1}(\psi(2)-\psi(1))=m(\psi(2)-\psi(1))=\frac{m}{1+\epsilon} \psi(1) .
$$


Hence

$$
\psi(m)=\frac{m-1}{1+\epsilon} \psi(1)+\psi(1)=\frac{1}{24}\left(1+\epsilon^{-1} m\right) .
$$

So

$$
\varphi(m)=\frac{1}{24} m(1-\epsilon m)\left(1+\epsilon^{-1} m\right)
$$

and then

$$
\omega(m, n)=\varphi(-n) \delta_{m+n, 0}=\frac{1}{24}\left(n^{3}-n-\left(\epsilon-\epsilon^{-1}\right) n^{2}\right) \delta_{m+n, 0}
$$

Moreover, it is easy to know that $\omega(m, n)$ satisfies equations (4.5)-(4.6).

Theorem 4.2. There does not exist a central extension of $V^{\beta, \epsilon}$ satisfying (4.5)-(4.6).

Proof. Firstly, let $m=-n \neq 0$ and $l=0$ in equation (4.6), we can get

$$
\omega(0,0)=\frac{1}{24} k\left(n^{2}-1\right)
$$

Since $\omega(0,0)$ does not depend on $n$, we know that $k=0$ and $\omega(0,0)=0$. Let $n=l=0$ in equations (4.5) and (4.6), we have

$$
\omega(m, 0)=\omega(0, m)=0
$$

On the other hand, let $m=0$ in equation (4.6), then we have

$$
n \omega(n, l)=\omega(0, n+l) f(n, l)-\omega(n, l) f(0, l)=-l \omega(n, l),
$$

that is, $(n+l) \omega(n, l)=0$. So we can assume that

$$
\omega(n, l)=\varphi(n) \delta_{n+l, 0} \text { for some map } \varphi: \mathbb{Z} \rightarrow \mathbb{C}
$$

Finally, let $m+n=0$ in equation (4.5), thus we get

$$
\varphi(n)-\varphi(-n)=\frac{1}{12}\left(-n^{3}+n\right) .
$$

So $\varphi(1)-\varphi(-1)=0$. Let $m, n \neq 0, m+n+l=0$ in equation (4.6), thereby we know

$$
(n-m) \varphi(m+n)=(m+n)(\varphi(n)-\varphi(m)) .
$$

Let $m=2, n=-1$ and $m=-2, n=1$ in the above equation respectively, we know that

$$
\varphi(2)=\varphi(-2)=4 \varphi(1)
$$

Hence $\varphi(2)-\varphi(-2)=0$. However, on the other hand,

$$
\varphi(2)-\varphi(-2)=\frac{1}{12}\left(-2^{3}+2\right)=-\frac{1}{2},
$$

which is a contradiction. 
Corollary 4.3. Any compatible left-symmetric algebra structure on the Virasoro algebra $\mathscr{V}$ satisfying equation (4.4) is isomorphic to one of the algebras given by the multiplication

$$
x_{m} x_{n}=\frac{n(1+\epsilon n)}{1+\epsilon(m+n)} x_{m+n}+\frac{\theta}{24}\left(n^{3}-n-\left(\epsilon-\epsilon^{-1}\right) n^{2}\right) \delta_{m+n, 0},
$$

where $\theta$ is an annihilator and $\operatorname{Re} \epsilon>0, \epsilon^{-1} \notin \mathbb{Z}$ or $\operatorname{Re} \epsilon=0, \operatorname{Im} \epsilon>0$.

Remark 4.4. The equation (4.8) was also obtained in $[\mathrm{Ku} 2]$ as a central extension of the left-symmetric algebra $V_{0, \epsilon}$ for $\epsilon \neq 0, \epsilon^{-1} \notin \mathbb{Z}$, which was given only as a special case of the left-symmetric algebras satisfying equation (3.1). It is interesting to see that in our discussion there does not exist a central extension satisfying equations (4.5)-(4.6) in other cases.

\section{ACKNOWLEDGEMENTS}

The authors thank Professor S.B. Tan for valuable discussions. This work was supported by the National Natural Science Foundation of China (10571091,10621101), NKBRPC (2006CB805905), Program for New Century Excellent Talents in University.

\section{REFERENCES}

[AS] A. Andrada, S. Salamon, Complex product structure on Lie algebras, Forum Math. 17 (2005) 261-295.

[Ba] C.M. Bai, A further study on non-abelian phase spaces: Left-symmetric algebraic approach and related geometry, Rev. Math. Phys. 18 (2006) 545-564.

[BK] B. Bakalov, V. Kac, Field algebras, Int. Math. Res. Not. (2003) 123-159.

[BN] A.A. Balinskii, S.P. Novikov, Poisson brackets of hydrodynamic type, Frobenius algebras and Lie algebras, Soviet Math. Dokl. 32 (1985) 228-231.

[Bo] M. Bordemann, Generalized Lax pairs, the modified classical Yang-Baxter equation, and affine geometry of Lie groups, Comm. Math. Phys. 135 (1990) 201-216.

[Bu1] D. Burde, Simple left-symmetric algebras with solvable Lie algebra, Manuscripta Math. 95 (1998) $397-411$.

[Bu2] D. Burde, Left-symmetric algebras, or pre-Lie algebras in geometry and physics, Cent. Eur. J. Math. 4 (2006) 323-357.

[Ca] A. Cayley, On the theory of analytic forms called trees, Collected Mathematical Papers of Arthur Cayley, Cambridge Univ. Press, Vol. 3 (1890) 242-246.

[Cha] F. Chapoton, Classification of some simple graded pre-Lie algebras of growth one, Comm. Algebra 32 (2004), 243-251.

[CL] F. Chapoton, M. Livernet, Pre-Lie algebras and the rooted trees operad, Int. Math. Res. Not. (2001) 395-408.

[Chu] B.Y. Chu, Symplectic homogeneous spaces, Trans. Amer. Math. Soc. 197 (1974) 145-159.

[CK] A. Connes, D. Kreimer, Hopf algebras, renormalization and noncommutative geometry, Comm. Math. Phys. 199 (1998) 203-242.

[DaM1] J.M. Dardie, A. Medina, Double extension symplectique d'un groupe de Lie symplectique, Adv. Math. 117 (1996) 208-227.

[DaM2] J.M. Dardie, A. Medina, Algebres de Lie kähleriennes et double extension, J. Algebra 185 (1996) $774-795$.

[DiM] A. Diatta, A. Medina, Classical Yang-Baxter equation and left-invariant affine geometry on Lie groups, Manuscripta Math. 114 (2004) 477-486.

[DL] A. Dzhumadil'daev, C. Lofwall, Trees, free right-symmetric algebras, free Novikov algebras and identities, Homology, Homotopy and applications 4 (2002) 165-190.

[E] K. Ebrahimi-Fard, Loday-type algebras and the Rota-Baxter relation, Lett. Math. Phys. 61 (2002) $139-147$.

[ES] P. Etingof, A. Soloviev, Quantization of geometric classical r-matrices, Math. Res. Lett. 6 (1999) $223-228$.

[GD] I.M. Gel'fand, I. Ya. Dorfman, Hamiltonian operators and algebraic structures related to them, Funct. Anal. Appl. 13 (1979) 248-262.

[G] M. Gerstenhaber, The cohomology structure of an associative ring, Ann. Math. 78 (1963) 267-288. 
XIAOLI KONG, HONGJIA CHEN, AND CHENGMING BAI

[GS] I.Z. Golubchik, V.V. Sokolov, Generalized operator Yang-Baxter equations, integrable ODEs and nonassociative algebras, J. Nonlinear Math. Phys. 7 (2000) 184-197.

[Ka] V. Kac, Infinite-dimensional Lie algebras, third edition, Cambridge Universitry Press, Cambridge (1990).

[KS] I. Kaplansy, L.J. Santharoubane, Harish-Chandra modules over the Virasoro algebra, Infinite-dimensional groups with applications, MSIR Publications, 4 (1985) 217-231.

[Ki] H. Kim, Complete left-invariant affine structures on nilpotent Lie groups, J. Diff. Geom. 24 (1986) 373-394.

[Ko] J.-L. Koszul, Domaines bornés homogènes et orbites de groupes de transformations affines, Bull. Soc. Math. France 89 (1961) 515-533.

[Ku1] B.A. Kupershmidt, Non-abelian phase spaces, J. Phys. A: Math. Gen. 27 (1994) 2801-2809.

[Ku2] B.A. Kupershmidt, On the nature of the Virasoro algebra, J. Nonlinear Math. Phy. 6 (1999) $222-245$.

[Ku3] B.A. Kupershmidt, What a classical $r$-matrix really is, J. Nonlinear Math. Phy. 6 (1999) 448-488.

[LL] J. Lepowsky, H.S. Li, Introduction to vertex operator algebras and their representations, Progress in Mathematics 227, Birkäuser, Boston (2004).

[LM] A. Lichnerowicz, A. Medina, On Lie groups with left invariant symplectic or kahlerian structures, Lett. Math. Phys. 16 (1988) 225-235.

[MP1] C. Martin, A. Piard, Indecomposable modules over the Virasoro Lie algebra and a conjecture of V. Kac, Comm. Math. Phys. 137, (1991) 109-132.

[MP2] C. Martin, A. Piard, Classification of the indecomposable bounded admissible modules over the Virasoro Lie algebra with weightspaces of dimension not exceeding two, Comm. Math. Phys. 150, (1992) 465-493.

[Ma1] O. Mathieu, Sur un problème de V.G.Kac: la classification de certaines algèbres de Lie gradues simples, J. Algebra, 102 (1986) 505-536.

[Ma2] O. Mathieu, Classification des algèbres de Lie graduées simples de croissance $\leq 1$, Invent. Math. 86 (1986) 371-426.

[Ma3] O. Mathieu, Classification of simple graded Lie algebras of finite growth, Invent. Math. 108 (1992) 445-519.

[Ma4] O. Mathieu, Classification of Harish-Chandra modules over the Virasoro Lie algebra, Invent. Math. 107 (1992) 225-234.

[Me] A. Medina, Flat left-invariant connections adapted to the automorphism structure of a Lie group, J. Diff. Geom. 16 (1981) 445-474.

[O] J.M. Osborn, Infinite dimensional Novikov algebras of characteristic 0, J. Algebra 167 (1994) $146-167$.

[SS] S.I. Svinolupov, V.V. Sokolov, Vector-matrix generalizations of classical integrable equations, Theoret. and Math. Phys. 100 (1994) 959-962.

[V] E.B. Vinberg, Convex homogeneous cones, Transl. of Moscow Math. Soc. No. 12 (1963) 340-403.

Xiaoli Kong, School of Mathematical Sciences, Xiamen University, Xiamen, Fujian 361005, P.R. CHINA

E-mail address: kongxl.math@gmail.com

Honguia Chen, Department of Mathematics, University of Science and Technology of China, Anhui, Hefei 230026, P.R. China

E-mail address: hjchen@mail.ustc.edu.cn

Chengming Bai, Chern Institute of Mathematics and LPMC, Nankai University, Tianjin 300071 , P.R.CHINA

E-mail address: baicm@nankai.edu.cn 